\title{
Modified fronto-lateral laryngectomy in treatment of glottic T1(a-b) cancer with anterior commissure involvement
}

\author{
Francesco Dispenza $^{\mathrm{a}, *}$, Carmelo Saraniti $^{\mathrm{b}}$, Navneet Mathur ${ }^{\mathrm{c}}$, Carlo Dispenza $^{\mathrm{b}}$ \\ ${ }^{a}$ Dipartimento Discipline Chirurgiche e Oncologiche U.O. Otorinolaringoiatria, Università degli Studi di Palermo, Palermo, Italy \\ ${ }^{\mathrm{b}}$ Dipartimento Neuroscienze Cliniche U.O. Otorinolaringoiatria, Università degli Studi di Palermo, Italy \\ ${ }^{\mathrm{c}}$ SK Soni Hospital, Jaipur, India
}

Received 18 May 2009; accepted 19 November 2009

Available online 23 December 2009

\begin{abstract}
Objective: Evaluation of clinical and oncological safety of the modified fronto-lateral laryngectomy in the treatment of T1a-b glottic cancer. Methods: Retrospective review of charts of patients managed with classical fronto-lateral laryngectomy or with our modified technique using a cervical fascia flap and a false cord flap to reconstruct the defect.

Results: No recurrence of cancer was observed in the present series and slight dysphonia was present in all cases. The patients managed with classical technique required a revision surgery for granulations or anterior synechia in 4 cases; those managed with modified technique did not need a second intervention.

Conclusion: The fronto-lateral laryngectomy should be present in the head-neck surgeon armamentarium. In T1a-b glottic cancer this technique gives a good oncological resection, but the postoperative period requires an intensive rehabilitation process. The modification of the classical technique reduces the incidence of a second intervention.
\end{abstract}

(C) 2009 Elsevier Ireland Ltd. All rights reserved.

Keywords: Laryngectomy; Fronto-lateral laryngectomy; Glottic cancer; Larynx surgery; Vertical laryngectomy

\section{Introduction}

In the management of laryngeal cancer the choice of the conservative surgical technique depends upon the TNM staging, tumor location and laryngeal structures involved. The anterior commissure (AC) of the glottis is a "risk region" for two reasons: its anatomical structure and the difficulty in delineating the deep extension of the tumor, even with the help of radiological study. Anatomically the AC constitutes thin mucosa with absence of internal perichondrium of thyroid cartilage along with proximity of the crico-thyroid membrane and of the caudal end of the epiglottis. This may erroneously lead to clinical down staging of the tumor, as $\mathrm{cT} 1$, which after histopathological examination turns out to be pT4.

\footnotetext{
* Corresponding author. Mobile: +39 3334565471.

E-mail address: francesco.dispenza@gmail.com (F. Dispenza).
}

The role of the AC in the development of the tumor is a matter of debate. Several authors consider the AC as a low resistance area to neoplastic growth [1-4]. Others authors suppose that $\mathrm{AC}$ is a barrier (wall) against anterior spreading of the tumor in the early phases of the tumoral growth $[5,6]$.

The Broyles's tendon acts as a barrier against the deep infiltration of the tumor in the early phases, but it does not restrict superficial spread either in the supraglottic or subglottic direction.

Carcinoma involving the $\mathrm{AC}$ is more aggressive than carcinoma arising in other sites, with the consequent worse prognosis. This observation led Fini-Storchi to add an anatomical-oncological classification to TNM staging of neoplasm involving the AC [7].

We present our oncologic and functional results in the patients affected by laryngeal cancer involving the AC treated with a modified frontal-lateral laryngectomy (FLL), a surgical procedure described by Leroux-Robert 
in 1956 [8] and popularized in Europe in 1970s [9], that today may be helpful in selected cases not well managed with endoscopic laser microsurgery or horizontal partial laryngectomies.

\section{Materials and methods}

A retrospective chart review of patients treated for laryngeal cancer with frontal-lateral laryngectomy in our department from 1994 to 2000 was conducted. History and symptoms were recorded in all cases. All patients underwent complete head and neck check up, including upper aerodigestive endoscopic examination. A computed tomography with contrast enhancement of the neck and standard X-ray chest projection were done in all patients. Patients were classified in accordance with TNM staging system. Biopsy of the laryngeal lesion was done under direct microlaryngoscopy in general anesthesia followed by FLL. Modified fronto-lateral technique was performed in about half of the patients since 1997, when we planned and introduced the variation from the classical technique. Complete information including all treatment options (i.e. surgery and radiation therapy) was provided to all patients and informed consent was obtained.

Surgical technique: the modification of the LerouxRobert technique consists of two additional surgical steps: (1) the use of a superficial fascia flap, dissected at the beginning of the operation, to reconstruct the defect due to excision of thyroid cartilage (Figs. 1 and 2) and (2) a shifting flap of false cord to reconstruct the glottic defect (Fig. 3).

In all cases temporary tracheostomy was performed and a naso-gastric tube was positioned. The voice rehabilitation was done after tracheostomy closure by speech-language pathologist for a period of 3 months.

The minimum follow-up period was of 5 years. It was accomplished with endoscopic evaluation of larynx and head and neck clinical examination at 1, 2, 3, 6, and 9 months in the first year after operation, every 6 months in the second and third year and once every year thereafter.
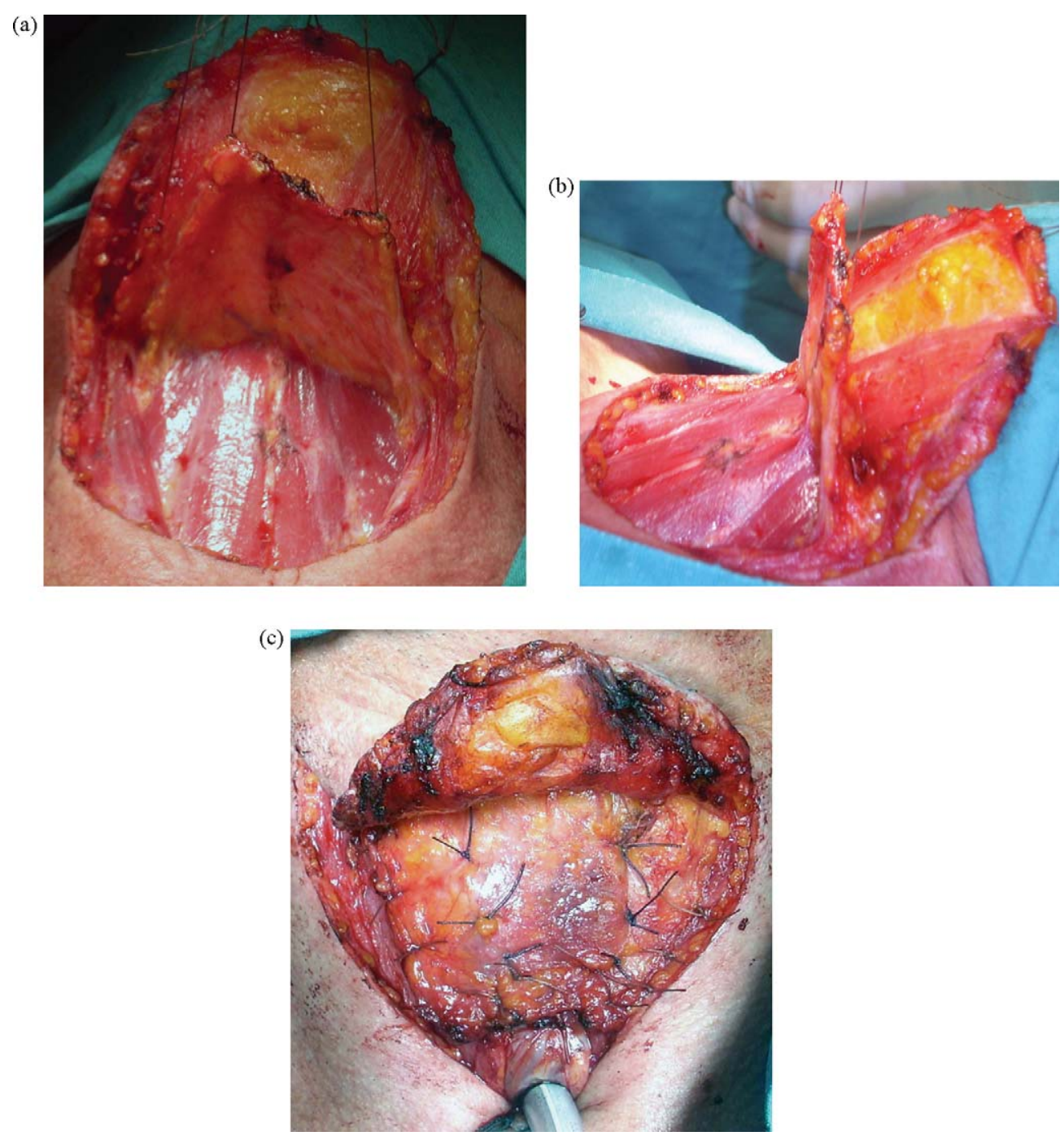

Fig. 1. The cervical fascia flap used in the reconstruction of anterior aspect of the larynx: (a) anterior view; (b) lateral view; (c) final reconstructive suture. 


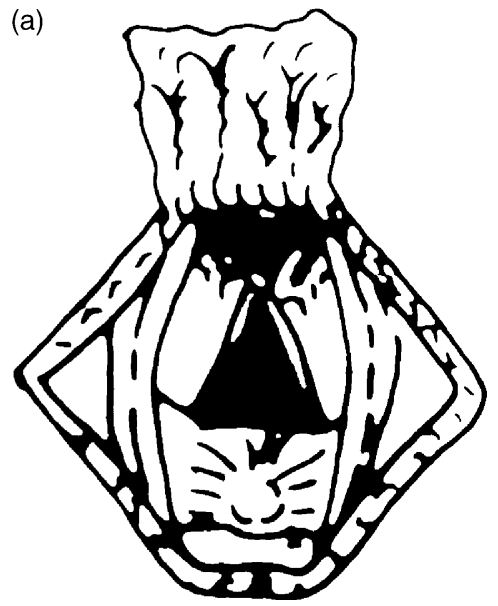

(b)

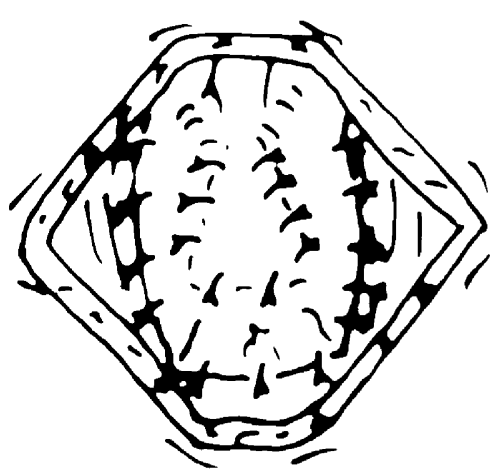

Fig. 2. Scheme of the cervical fascia flap suture: (a) the thyroid cartilage gap created after tumor removal will be covered by the cervical fascia flap elevated as first step of the operation; (b) the suture of the flap to the cartilage border and to the strap muscles, over the thyroid cartilage gap.

Our Institutional Review Board approved the present study.

\section{Results}

Twenty-one male patients with fronto-lateral laryngectomy were treated in our Department, from 1994 to 2000, to
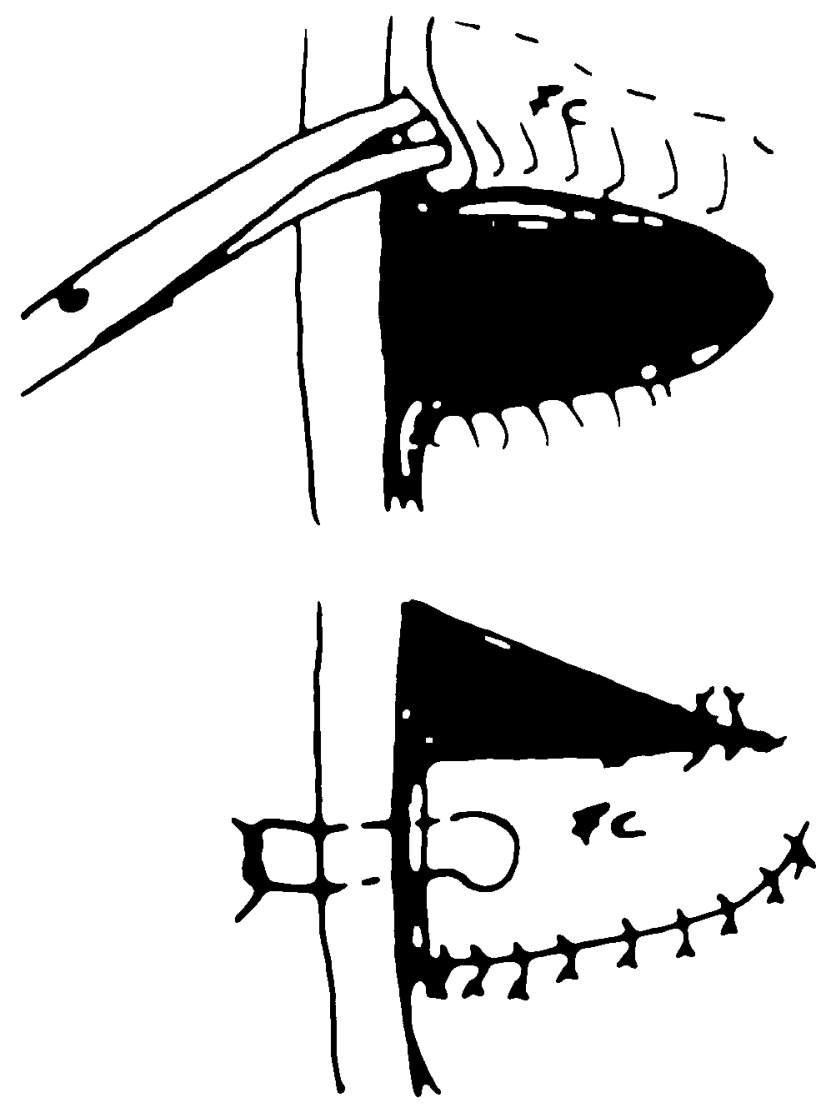

Fig. 3. Scheme of the false cord flap to reconstruct the glottic plane after tumor removal; the flap is dissected and rotated downward to fill the glottic plane gap and then sutured. manage glottic cancer extending to the AC. All patients who underwent surgery refused radiation therapy as treatment. The mean age was 64 years (range 53-75 years). All patients were heavy smokers: 6 patients more than 20 cigarettes per day and 15 patients more than 30 . In 13 cases more than $200 \mathrm{ml}$ of daily alcohol consumption was associated with smoking. The symptoms recorded were: dysphonia in 21 cases, productive cough in 7 cases and dry in 3 patients, pharyngeal discomfort in 4 patients. The biopsy showed an infiltrating squamous cell carcinoma in all cases. The Leroux-Robert's fronto-lateral laryngectomy was performed in 8 cases until March 1997. Since June 1997 to December 2000 we performed the modified technique described above in 13 patients.

The definitive histopathological examination confirmed the presence of a squamous cell carcinoma in all series. In 5 $(23 \%)$ out of 21 patients an early thyroid cartilage invasion was present, but always contained by the cartilage.

The naso-gastric tube was positioned for an average of 6 days (range 4-13 days). The deglutition was normal in all cases. The tracheostomy tube was kept for less than 10 days in 10 patients, between 10 and 20 days in 10 patients and 25 days in one case.

The mean follow-up was 9.5 years (range $7-15$ years).

All patients presented with slight dysphonia. In 4 patients treated with the classical Leroux-Robert technique a revision microlaryngoscopic surgery was necessary to remove glottic granulations in 3 patients and anterior synechia in 1 (Fig. 4). In the patients managed with the modified fronto-lateral technique we noted glottic granulations only in one case. All patients are without recurrence till date (Fig. 5).

\section{Discussion}

The surgical treatment of T1a-b glottic cancer with AC involvement, extending to the anterior third of the 


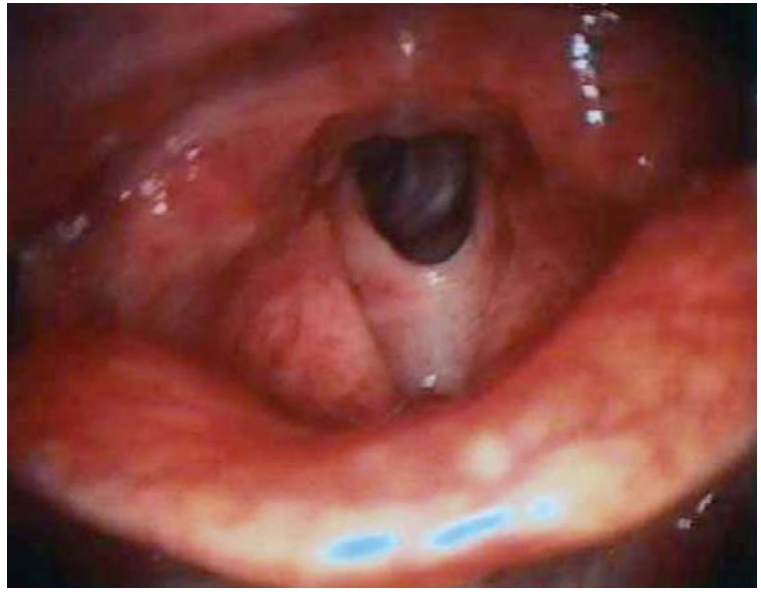

Fig. 4. Anterior synechia in a patient treated with the classical LerouxRobert technique.

contralateral vocal cord, has two main objectives: (1) oncologically safe excision; (2) sparing of laryngeal functions. The less invasive techniques, such as endoscopic laser microsurgical approach, is often preferred because of few postoperative complications and it allows preservation of thyroid cartilage integrity thus leaving a natural barrier in case of recurrence.

Sometimes doubt exists about the deep extension of cancer involving the AC toward the thyroid cartilage. In this case choice of a less invasive technique (transoral surgery) may not be appropriate, while a supracricoid partial laryngectomy as cricohyoidoepiglottopexy may represent an overtreatment; therefore in our view the use of the vertical partial surgery such as the FLL offers a more effective control of the disease, with preservation of laryngeal function in case of only one vocal cord and AC involvement.

Leroux-Robert demonstrated that the FLL permits the excision of the vocal cord, the correspondent subglottic area, $\mathrm{AC}$ and subcommissural region. The technique includes resection of the dihedral angle of thyroid cartilage together with the lesion. According to Leroux-Robert the indication of FLL is a cancer that involves the vocal cord from the arytenoid cartilage to the $\mathrm{AC}$, even if the motility of such a cord is impaired.

The Leroux-Robert's indication enjoyed wide popularity among several authors [3,10-13]. Other authors adopted the FLL in the vocal cord carcinoma that crosses the midline in the region of $\mathrm{AC}$ [14].

The posterior extension toward the arythenoid cartilage is not an absolute contraindication to perform FLL. De Campora and Radici limit the FLL application to vocal cord cancer that does not involve the vocal process of the arythenoid cartilage and disapproves this technique also in cases with contralateral vocal cord involvement, subglottic and ventricular extension [3].

The postoperative period after FLL requires hospitalization for about 6 days because of tracheostomy tube management and naso-gastric tube feeding. Tracheostomy tube was needed for a mean period of 11 days (only one case needed it for 25 days), until the healing of glottis was considered safe for breathing. It is noticeable that Brumund et al. reported a large series of patients treated with FLL without performing a temporary tracheostomy and without naso-gastric tube positioning. However, our data of postoperative care are in accordance with the length of time previously reported in literature $[9,15,16]$.

The voice quality after a classic FLL is similar to that obtained after a cordectomy via laryngofissure. The anterior synechia seems to have a significant impact on the voice quality in patients treated with FLL [17]. As suggested by some authors, the reconstruction of the glottis with a false cord flap results in a better phonatory outcome, because of reduction of the risk of anterior synechia [18,19]. The cervical fascia or mucochondral nasal septum free flap [20] gives a good support to the pre-laryngeal tissues, avoiding its herniation in the laryngeal space, and facilitating the glottic healing (Fig. 5). In our experience the cervical fascia and the false cord flap give a good functional result and for this reason we adopted such technique in all patients since 1997.

The rate of complications in our patients was low. No patients had infection of wound or fistula formation. The modification of the classical technique allowed, in our
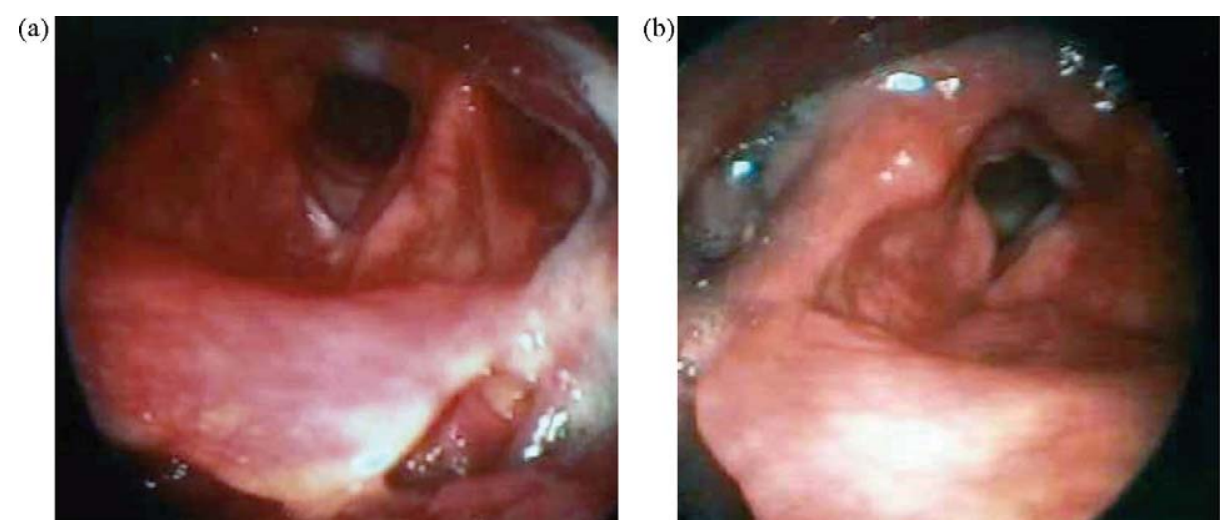

Fig. 5. Postoperative examination of cases treated with our modified fronto-lateral laryngectomy, the triangular shape of the new glottic plane obtained with suggested modifications is noticeable. 
series, a drastic reduction of laryngeal granulation development in the postoperative period.

We did not observe any problem in swallowing in our series of patients; the nasal-gastric tube was removed in all patients in about 6 days from operation.

The absence of local recurrence in our series is probably due to strict selection of patients; we performed FLL only in patients with a glottic cancer T1a-b with AC involvement. Fiorella et al. showed that FLL was not sufficient for advanced tumor involving AC [13]. We usually advocate a supracricoid partial laryngectomy (i.e. cricohyoidoepiglottopexy) for glottic T2 stage, because of high risk of recurrence noted in the other centre [21,22].

In the last decade we performed less FLL due to practice of endoscopic $\mathrm{CO}_{2}$ laser surgery that permitted excision of T1a-b glottic tumors. We reserve the modified FLL for patients with $\mathrm{AC}$ involvement who have contraindication to laser surgery, inadequate laryngeal exposure for endoscopic surgery, and for patients with low performance status in which reconstructive laryngectomy procedures are contraindicated.

\section{Conclusions}

Head and neck surgeons should consider fronto-lateral laryngectomy for selected patients that choose surgical therapy and are not well manageable with $\mathrm{CO}_{2}$ laser surgery. In $\mathrm{T} 1 \mathrm{a}-\mathrm{b}$ glottic tumor with $\mathrm{AC}$ involvement this technique gives a reasonable oncological safe resection. The recurrence rate is comparable with other procedures for T1a-b glottic cancer, but the postoperative period is longer and needs an intensive rehabilitation process. From the time when we adopted the modified FLL we observed optimal results in disease treatment and functional outcome. The modified technique reduces the postoperative risk of synechia and granulation tissue development thus improving the quality of the voice.

\section{Conflict of interest}

None.

\section{References}

[1] Harrison DF. Significance and means by which laryngeal cancer invades thyroid cartilage. Ann Otol Rhinol Laryngol 1984;93(JulyAugust (4 Pt 1)):293-6.

[2] Rucci L, Romagnoli P, Casucci A, Ferlito A. Embryological study of the glottic site and clinical implications. Oral Oncol 2004;40(November (10)):1017-25.
[3] De Campora E, Radici M. La commissura anteriore nell'evoluzione dei tumori del piano glottico: considerazioni classificative, prognostiche e terapeutiche. Acta Otolaryngol Ital 1994;14:143-55.

[4] Sessions DG, Ogura JH, Fried MP. The anterior commissure in glottic carcinoma. Laryngoscope 1975;85(October (10)):1624-32.

[5] Kirchner JA, Fifteenth Daniel C, Baker Jr. Memorial lecture. What have whole organ sections contributed to the treatment of laryngeal cancer? Ann Otol Rhinol Laryngol 1989;98(September (9)):661-7.

[6] Tucker Jr GF, Alonso WA, Cowan M, Tucker JA, Druck N. The anterior commissure revisited. Ann Otol Rhinol Laryngol 1973; 82(September-October (5)):625-36.

[7] Fini-Storchi O, Rucci L, Gallo O, Trovati M. Coinvolgimento neoplastico della commessura anteriore. In: Paper Presented at: LXXX Congresso Nazionale della Società Italiana di otorinolaringoiatria; 1993.

[8] Leroux-Robert J. Indications for a radical surgery, partial surgery, radiotherapy and combined surgery and radiotherapy for cancer of the larynx and hypopharynx. Ann Otol Rhinol Laryngol 1956;65:137-53.

[9] Leroux-Robert J. A statistical study of 620 laryngeal carcinomas of the glottic region personally operated upon more than five years ago. Laryngoscope 1975;85:1440-52.

[10] Alajmo E, Fini-Storchi O, Polli G. Five-year results of 1000 patients operated on for cancer of the larynx. Acta Otolaryngol Nov-Dec 1976;82(5-6):437-9.

[11] Alajmo E. Role of reconstructive laryngectomy in larynx surgery. Rev Laryngol Otol Rhinol (Bord) 1976;97(May-June (5-6)):207-10.

[12] Fini Storchi O, Rucci L, Agostini V. Indication for fronto-lateral laryngectomy. Critical evaluation of the course of 49 cases. Acta Otorhinolaryngol Ital 1981;1(July-August (1)):95-9.

[13] Fiorella R, Di Nicola V, Mangiatordi F, Fiorella ML. Indications for frontolateral laryngectomy and prognostic factors of failure. Eur Arch Otorhinolaryngol 1999;256(8):423-5.

[14] Piquet JJ. La chirurgie reconstructive. In: Portman M, Guerrier Y, editors. Technique chirurgicale ORL et Cervico-Faciale, vol. 3. Paris: Masson; 1997. p. 321-8.

[15] Olsen KD, DeSanto LW. Partial vertical laryngectomy-indications and surgical technique. Am J Otolaryngol 1990;11:153-60.

[16] Apostolopoulos K, Samaan R, Labropoulou E. Experience with vertical partial laryngectomy with special reference to laryngeal reconstruction with cervical fascia. J Laryngol Otol 2002;116:19-23.

[17] Dedivitis RA, Queija DS, Barros AP, Pfuetzenreiter Jr EG, Felippu A, Salvia VF, et al. The impact of the glottic configuration after frontolateral laryngectomy on the perceptual voice analysis: a preliminary study. J Voice 2008;22(November (6)):760-4.

[18] Brumund KT, Gutierrez-Fonseca R, Garcia D, Babin E, Hans S, Laccourreye O. Frontolateral vertical partial laryngectomy without tracheotomy for invasive squamous cell carcinoma of the true vocal cord: a 25year experience. Ann Otol Rhinol Laryngol 2005;114(April (4)):314-22.

[19] Biacabe B, Crevier-Buchman L, Hans S, Laccourreye O, Brasnu D. Phonatory mechanism after vertical partial laryngectomy with glottic reconstruction by false vocal fold flap. Ann Otol Rhinol Laryngol 2001;110:935-40.

[20] Szmeja Z, Szyfter W, Wojtowicz JG, Kruk-Zagajewska A. Extended fronto-lateral laryngectomy with simultaneous reconstruction by means of a mucochondral nasal septum flap. Eur Arch Otorhinolaryngol 1999;256(8):390-1.

[21] Peretti G, Nicolai P, Redaelli De Zinis LO, Berlucchi M, Bazzana T, Bertoni $\mathrm{F}$, et al. Endoscopic $\mathrm{CO}_{2}$ laser excision for Tis, T1 and T2 glottic carcinomas: cure rate and prognostic factors. Otolaryngol Head Neck Surg 2000;123:124-31.

[22] Eckel HE, Thumfart W, Jungehulsing M, Sittel C, Stenert E. Transoral laser surgery for early glottic carcinoma. Eur Arch Otolaryngol 2000; 257:221-6. 